P

Noberef tuluer

Journal of Nonlinear Mathematical Physics

ISSN (Online): 1776-0852 ISSN (Print): 1402-9251

Journal Home Page: https://www.atlantis-press.com/journals/jnmp

Some extensions on the soliton solutions for the Novikov equation with cubic nonlinearity

Chaohong Pan, Yating Yi

To cite this article: Chaohong Pan, Yating Yi (2015) Some extensions on the soliton solutions for the Novikov equation with cubic nonlinearity, Journal of Nonlinear Mathematical Physics 22:2, 308-320, DOI:

https://doi.org/10.1080/14029251.2015.1033243

To link to this article: https://doi.org/10.1080/14029251.2015.1033243

Published online: 04 January 2021 


\title{
Some extensions on the soliton solutions for the Novikov equation with cubic nonlinearity
}

\author{
Chaohong Pan \\ Department of Mathematics, South China University of Technology, \\ Guangzhou, 510640, China \\ pan.ch@mail.scut.edu.cn \\ Yating Yi \\ Department of Mathematics, South China University of Technology, \\ Guangzhou, 510640, China \\ yi.yating@mail.scut.edu.cn
}

Received 23 September 2014

Accepted 6 March 2015

\begin{abstract}
In this paper, by using the bifurcation method of dynamical systems, we derive the traveling wave solutions of the nonlinear equation $U U_{\tau y y}-U_{y} U_{\tau y}+U^{2} U_{\tau}+3 U_{y}=0$. Based on the relationship of the solutions between the Novikov equation and the nonlinear equation, we present the parametric representations of the smooth and nonsmooth soliton solutions for the Novikov equation with cubic nonlinearity. These solutions contain peaked soliton, smooth soliton, W-shaped soliton and periodic solutions. Our work extends some previous results.

Keywords: the Novikov equation; smooth and nonsmooth solitons; traveling wave solutions; bifurcation method.
\end{abstract}

2000 Mathematics Subject Classification: 76M60, 58D19, 35D30

\section{Introduction}

In this paper, we consider the Novikov equation [1]

$$
m_{t}+u^{2} m_{x}+3 u u_{x} m=0, \quad m=u-u_{x x} .
$$

Eq. (1.1) was introduced to classify the nonlocal partial differential equations(PDEs). By using the perturbative symmetry approach, some symmetries and a scalar Lax representation of Eq. (1.1) are provided in [1]. Further, Hone and Wang [2] presented the matrix Lax representation, the biHamiltonian structure, an infinite number of conservation laws and established the relationship between the Novikov equation and the Sawada-Kotera equation by a reciprocal transformation. As $\mathrm{Li}$ and Liu [3] stated, Eq. (1.1) is the Camassa-Holm type equation with cubic nonlinearity and they also introduced the two-component Novikov equation with a bi-Hamiltonian structure. As a generalization of the Novikov equation, Geng and Xue [4] studied a coupled model with cubic nonlinearity.

A remarkable feature of the Novikov equation is that it admits peakons whose dynamics are shown to obey a finite dimensional integrable Hamiltonian system $[2,5,6]$. Moreover, by using a 
reciprocal transformation, Matsuno [7] presented a nonlinear equation

$$
U U_{\tau y y}-U_{y} U_{\tau y}+U^{2} U_{\tau}+3 U_{y}=0
$$

which was introduced to construct a compact parametric representation of the smooth bright multisoliton solutions on a constant background for the Novikov equation. The parametric representation for the multisoliton solution is of the form

$$
\begin{aligned}
u^{2} & =u^{2}(y, \tau)=\kappa^{3}+\frac{1}{2} \frac{\partial}{\partial \tau} \ln \frac{g_{1}}{g_{2}}, \\
x & =x(y, \tau)=\frac{y}{\kappa}+\kappa^{3} \tau+\frac{1}{2} \ln \frac{g_{1}}{g_{2}}+d,
\end{aligned}
$$

where the parameters $\kappa, d$ and the tau-functions $g_{1}, g_{2}$ are given in [7].

In particular, $\mathrm{Li}$ [8] used the bifurcation method of dynamical systems to study the parametric representations of cuspon and compacton solutions of Eq. (1.1) and discuss their dynamical behavior. By introducing the variable $\chi$ and making the transformation $\psi=\frac{u}{\sqrt{u^{2}-c}}$, the cuspon is of the form

$$
\begin{aligned}
\psi(\chi)= & \psi_{1}-\left(\psi_{1}-\psi_{0}\right) \operatorname{coth}^{2} \chi \\
u(\chi)= & \frac{\sqrt{c} \psi(\chi)}{\sqrt{\psi(\chi)^{2}-1}}, \\
\xi(\chi)= & \pm \frac{c}{\sqrt{A}}\left[\frac{-2}{\left(\psi_{0}^{2}-1\right) \sqrt{\psi_{1}-\psi_{0}}} \chi+\Psi_{0}\right. \\
& \left.+\frac{\ln \left(\frac{\sqrt{\psi_{1}-1}+\sqrt{\psi_{1}-\psi(\chi)}}{\sqrt{\psi_{1}-\psi(\chi)}-\sqrt{\psi_{1}-1}}\right)}{2\left(\psi_{0}-1\right) \sqrt{\psi_{1}-1}}-\frac{\ln \left(\frac{\sqrt{\psi_{1}+1}+\sqrt{\psi_{1}-\psi(\chi)}}{\sqrt{\psi_{1}-\psi(\chi)}-\sqrt{\psi_{1}+1}}\right)}{2\left(\psi_{0}+1\right) \sqrt{\psi_{1}+1}}\right],
\end{aligned}
$$

and the compacton is of the form

$$
\begin{aligned}
\psi(\chi)= & -1+\frac{\psi_{2}-\psi_{3} \operatorname{sn}^{2}(\chi, k)}{\operatorname{cn}^{2}(\chi, k)}, \\
u(\chi)= & \frac{\sqrt{c} \psi(\chi)}{\sqrt{\psi(\chi)^{2}-1}}, \\
\xi(\chi)= & -\frac{c}{\sqrt{A\left(\psi_{1}-\psi_{2}\right)}}\left[\frac{1}{\psi_{3}-1} \chi-\frac{1}{\psi_{3}+1} F(\arcsin (\operatorname{sn}(\chi, k)), k)\right. \\
& +\frac{\psi_{3}-\psi_{2}}{\left(\psi_{2}-1\right)\left(\psi_{3}-1\right)} \Pi\left(\arcsin (\operatorname{sn}(\chi, k)), \alpha_{1}^{2}, k\right) \\
& \left.-\frac{\psi_{3}-\psi_{2}}{\left(\psi_{2}+1\right)\left(\psi_{3}+1\right)} \Pi\left(\arcsin (\operatorname{sn}(\chi, k)), \alpha_{2}^{2}, k\right)\right],
\end{aligned}
$$

where the parameters are given in [8].

The purpose of this paper is to extend the smooth and nonsmooth soliton solutions of the Novikov equation. Firstly, we establish the relationship of the soliton solutions between the Novikov equation and Eq. (1.2) by a transformation formula. Secondly, based on the bifurcation method of dynamical systems [8-13], the exact traveling wave solutions of Eq. (1.2) are given. Lastly, we construct some novel soliton solutions and their parametric representations for Eq. (1.1). 
Notice that the relationship between the Novikov equation and some nonlinear equations has been discussed through the reciprocal transformation [7]. Here we turn our attention to the relationship of the soliton solutions between the Novikov equation and Eq. (1.2). This makes it possible to obtain exact soliton solutions of the Novikov equation easily. First, we recall some previous results and show them by two Lemmas as follows.

Lemma 1.1. (see [7]) In accordance with [2], using the coordinate transformation $(x, t) \longrightarrow(y, \tau)$ by

$$
d y=m^{\frac{2}{3}} d x-m^{\frac{2}{3}} u^{2} d t, d \tau=d t
$$

then the derivatives of $x$ and $t$ can be rewritten as

$$
\frac{\partial}{\partial x}=m^{\frac{2}{3}} \frac{\partial}{\partial y}, \frac{\partial}{\partial t}=\frac{\partial}{\partial \tau}-m^{\frac{2}{3}} u^{2} \frac{\partial}{\partial y},
$$

and the variable $x=x(y, \tau)$ satisfies a system of linear PDEs

$$
x_{y}=m^{-\frac{2}{3}}, x_{\tau}=u^{2}
$$

then the Novikov equation can be recast into the form

$$
m_{\tau}+3 m^{\frac{5}{3}} u u_{y}=0
$$

On the other hand, Eq. (1.1) can be rewritten in terms of $m$ as

$$
u=m+m^{\frac{4}{3}} u_{y y}+\frac{2}{3} m^{\frac{1}{3}} m_{y} u_{y} .
$$

Lemma 1.2. (see [7]) Define the variables $V$ and $W$ by

$$
V=m^{\frac{2}{3}}, W=u m^{\frac{1}{3}}
$$

then (1.9) and (1.10) can be put into the form (see [2])

$$
\begin{aligned}
& \left(\frac{1}{V}\right)_{\tau}=\left(\frac{W^{2}}{V}\right)_{y}, \\
& W_{y y}+U W+1=0,
\end{aligned}
$$

where

$$
U=-\frac{V_{y y}}{2 V}+\frac{V_{y}^{2}}{4 V^{2}}-\frac{1}{V^{2}}
$$

By adopting the linear PDE

$$
U_{\tau}+3 W_{y}=0
$$

eliminating the variable $W$ from (1.13) and (1.15), they obtained the equation Eq. (1.2). 
Corollary 1.1. Assume that a traveling wave solution of Eq. (1.2) is of the form

$$
U(y, \tau)=\varphi(\xi), \quad \xi=y-c \tau
$$

Combining (1.8), (1.11), (1.12) and (1.15), we get the parametric representations of the solutions of Eq. (1.1) as follows

$$
\left\{\begin{array}{l}
u^{2}=-g_{2}+\frac{g_{2}}{1+\frac{c}{9}\left(\varphi+\frac{g_{1}}{c}\right)^{2}}, \\
x=\int u^{2} d \tau
\end{array}\right.
$$

where $c$ is the wave speed, $g_{1}$ and $g_{2}$ are the integral constants.

We notice that the unbounded traveling wave solutions of Eq. (1.2) will change to the bounded soliton solutions of Eq. (1.1) by employing the formula (1.17). Therefore, we only need to consider the traveling wave solution of Eq. (1.2) instead of those of Eq. (1.1) where the cubic nonlinearity creates a great difficulty.

\section{Traveling wave solutions of Eq. (1.2)}

First, we substitute (1.16) into Eq. (1.2), it follows that

$$
-c \varphi \varphi^{\prime \prime \prime}+c \varphi^{\prime} \varphi^{\prime \prime}-c \varphi^{2} \varphi^{\prime}+3 \varphi^{\prime}=0 .
$$

Integrating (2.1) once, we have

$$
-c \varphi \varphi^{\prime \prime}+c \varphi^{\prime 2}-\frac{c}{3} \varphi^{3}+3 \varphi+g=0,
$$

where $g$ is the integral constant.

Letting $z=\frac{d \varphi}{d \xi}$, we get the following planar dynamic system

$$
\left\{\begin{array}{l}
\frac{d \varphi}{d \xi}=z \\
\frac{d z}{d \xi}=\frac{1}{c \varphi}\left(c z^{2}-\frac{c}{3} \varphi^{3}+3 \varphi+g\right) .
\end{array}\right.
$$

Assume that $d \xi=c \varphi d \mu$, so that (2.3) is equivalent to the system as follows:

$$
\left\{\begin{array}{l}
\frac{d \varphi}{d \mu}=c \varphi z \\
\frac{d z}{d \mu}=c z^{2}-\frac{c}{3} \varphi^{3}+3 \varphi+g,
\end{array}\right.
$$

which has the first integral

$$
H(\varphi, z)=z^{2}+\frac{2}{3} \varphi^{3}+\frac{6}{c} \varphi-h \varphi^{2}+\frac{g}{c} .
$$

In what following, we also assume that

$$
f(\varphi)=-\frac{c}{3} \varphi^{3}+3 \varphi+g .
$$

Then we have

$$
f^{\prime}(\varphi)=-c \varphi^{2}+3
$$


Solving $f^{\prime}(\varphi)=0$ for $c>0$, we get two roots as follows:

$$
\varphi_{0}^{-}=-\sqrt{\frac{3}{c}}, \quad \varphi_{0}^{+}=\sqrt{\frac{3}{c}} .
$$

By using the properties of equilibrium points and bifurcation theory, we obtain three bifurcation curves as follows:

$$
\begin{aligned}
& g_{1}(c)=-2 \sqrt{\frac{3}{c}}, \\
& g_{2}(c)=0, \\
& g_{3}(c)=2 \sqrt{\frac{3}{c}} .
\end{aligned}
$$
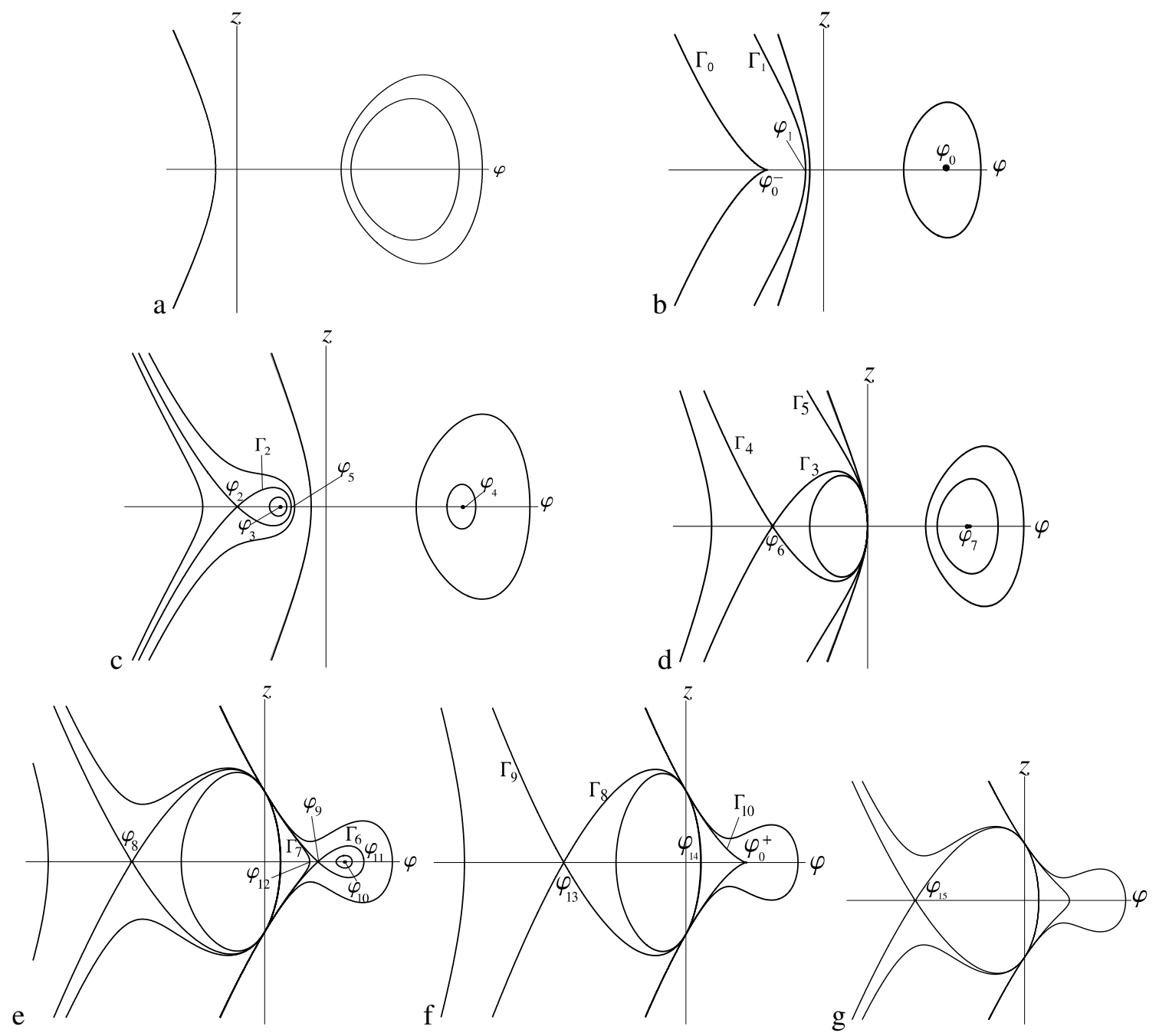

Fig. 1. The phase portraits of system (2.3) under some conditions. (a) $g>g_{3}(c)$; (b) $g=g_{3}(c)$; (c) $0<g<g_{3}(c)$; (d) $g=0$; (e) $g_{1}(c)<g<0$; (f) $g=g_{1}(c)$; (g) $g<g_{1}(c)$. 
Consequently, according to the qualitative theory, we obtain the bifurcation phase portraits for system (2.4) as Fig. 1.

Based on the dynamics of the level curves determined by $H(\varphi, z)=h$ and the bifurcation phase portraits of system (2.3), we will give the explicit expressions of the traveling wave solutions for Eq. (1.2), from which we obtain the parametric representations of the smooth and nonsmooth soliton solutions of Eq. (1.1) with the help of the formula (1.17).

First, we introduce some marks for simplicity. For given parameters $c$ and $g$, let

$$
\begin{aligned}
\varphi_{0} & =2 \sqrt{\frac{3}{c}} \\
\varphi_{1} & =-\sqrt{\frac{3}{16 c}}, \\
\varphi_{3} & =\frac{1}{2}\left(-\varphi_{2}-\sqrt{\varphi_{2}^{2}-\frac{12 g}{c \varphi_{2}}}\right), \\
\varphi_{4} & =\frac{1}{2}\left(-\varphi_{2}+\sqrt{\varphi_{2}^{2}-\frac{12 g}{c \varphi_{2}}}\right) \\
\varphi_{5} & =\frac{9-c \varphi_{2}^{2}}{2 c \varphi_{2}}, \\
\varphi_{6} & =-\sqrt{\frac{9}{c}}, \\
\varphi_{7} & =\sqrt{\frac{9}{c}} \\
\varphi_{9} & =\frac{1}{2}\left(-\varphi_{8}-\sqrt{\varphi_{8}^{2}-\frac{12 g}{c \varphi_{8}}}\right), \\
\varphi_{10} & =\frac{1}{2}\left(-\varphi_{8}+\sqrt{\varphi_{8}^{2}-\frac{12 g}{c \varphi_{8}}}\right), \\
\varphi_{11} & =\frac{-3 g}{2 c \varphi_{9}^{2}}, \\
\varphi_{12} & =\frac{-3 g}{2 c \varphi_{10}^{2}}, \\
\varphi_{13} & =-2 \sqrt{\frac{3}{c}}, \\
&
\end{aligned}
$$

where $\varphi_{6}<\varphi_{2}<\varphi_{0}^{-}$and $\varphi_{13}<\varphi_{8}<\varphi_{6}$.

The exact traveling wave solutions of Eq. (1.2) are listed in the following three cases.

Case 1. Eq. (1.2) possesses the unbounded solutions are of the expressions

$$
\varphi_{1}(\xi)=\varphi_{0}^{-}-\frac{6}{\xi^{2}}
$$




$$
\begin{gathered}
\varphi_{5}(\xi)=\varphi_{6} \operatorname{coth}^{2}\left(\sqrt{\frac{-\varphi_{6}}{6}} \xi\right) \\
\varphi_{10}(\xi)=\varphi_{14}-\left(\varphi_{14}-\varphi_{13}\right) \operatorname{coth}^{2} \frac{\eta_{5}}{2}, \text { where } \eta_{5}=\sqrt{\frac{2}{3}\left(\varphi_{14}-\varphi_{13}\right)},
\end{gathered}
$$

and

$$
\varphi_{11}(\xi)=\varphi_{0}^{+}-\frac{6}{\xi^{2}}
$$

Case 2. Eq. (1.2) possesses the periodic blow-up solutions are of the expressions

$$
\begin{gathered}
\varphi_{2}(\xi)=\varphi_{1}-\left(\varphi_{0}-\varphi_{1}\right) \tan ^{2} \frac{\eta_{1}}{2}, \text { where } \eta_{1}=\sqrt{\frac{2}{3}\left(\varphi_{0}-\varphi_{1}\right)} \xi \\
\varphi_{6}(\xi)=-\varphi_{7} \tan ^{2}\left(\sqrt{\frac{\varphi_{7}}{6}} \xi\right)
\end{gathered}
$$

and

$$
\varphi_{8}(\xi)=\varphi_{12}-\left(\varphi_{10}-\varphi_{12}\right) \tan ^{2} \frac{\eta_{4}}{2}, \text { where } \eta_{4}=\sqrt{\frac{2}{3}\left(\varphi_{10}-\varphi_{12}\right)} \xi
$$

Case 3. Eq. (1.2) possesses the solitary wave solutions are of the expressions

$$
\begin{gathered}
\varphi_{3}(\xi)=\varphi_{5}-\left(\varphi_{5}-\varphi_{2}\right) \tanh ^{2} \frac{\eta_{2}}{2}, \text { where } \eta_{2}=\sqrt{\frac{2}{3}\left(\varphi_{5}-\varphi_{2}\right)} \xi \\
\varphi_{4}(\xi)=\varphi_{6} \tanh ^{2}\left(\sqrt{\frac{-\varphi_{6}}{6}} \xi\right) . \\
\varphi_{7}(\xi)=\varphi_{11}-\left(\varphi_{11}-\varphi_{9}\right) \tanh ^{2} \frac{\eta_{3}}{2}, \text { where } \eta_{3}=\sqrt{\frac{2}{3}\left(\varphi_{11}-\varphi_{9}\right)} \xi
\end{gathered}
$$

and

$$
\varphi_{9}(\xi)=\varphi_{14}-\left(\varphi_{14}-\varphi_{13}\right) \tanh ^{2} \frac{\eta_{5}}{2} .
$$

The correctness of these solutions is tested as well by using the software Mathematica. We notice that some explicit solitons of this kind of equation as (1.2) can be obtained by the singular manifold method(see [14]). Next, we will give the demonstrations on Cases 1-3. 
(1) When $g=g_{3}(c)$, the curves $\Gamma_{0}$ and $\Gamma_{1}$ possess the following expressions:

$$
\begin{gathered}
\Gamma_{0}: z=\operatorname{sign}(z) \sqrt{\frac{2}{3}}\left(\varphi_{0}^{-}-\varphi\right) \sqrt{\varphi_{0}^{-}-\varphi}, \text { where } \varphi<\varphi_{0}^{-}, \\
\Gamma_{1}: \quad z=\operatorname{sign}(z) \sqrt{\frac{2}{3}}\left(\varphi_{0}-\varphi\right) \sqrt{\varphi_{1}-\varphi}, \text { where } \varphi<\varphi_{1} .
\end{gathered}
$$

Substituting (2.19) and (2.20) into $\frac{d \varphi}{d \xi}=z$ and integrating them along $\Gamma_{0}$ and $\Gamma_{1}$ respectively, we have

$$
\begin{gathered}
\int_{-\infty}^{\varphi} \frac{d s}{\left(\varphi_{0}^{-}-s\right) \sqrt{\varphi_{0}^{-}-s}}=\sqrt{\frac{2}{3}} \operatorname{sign}(z) \xi\left(\operatorname{along} \Gamma_{0}\right), \\
\int_{\varphi}^{\varphi_{1}} \frac{d s}{\left(\varphi_{0}-s\right) \sqrt{\varphi_{1}-s}}=-\sqrt{\frac{2}{3}} \operatorname{sign}(z) \xi\left(\operatorname{along} \Gamma_{1}\right) .
\end{gathered}
$$

In (2.21) and (2.22) completing the integrals yields (2.8) and (2.12) respectively.

(2) When $0<g<g_{3}(c)$, the curve $\Gamma_{2}$ possesses the following expression:

$$
z=\operatorname{sign}(z) \sqrt{\frac{2}{3}}\left(\varphi-\varphi_{2}\right) \sqrt{\varphi_{5}-\varphi}, \text { where } \varphi_{2}<\varphi \leq \varphi_{5}
$$

Substituting (2.23) into $\frac{d \varphi}{d \xi}=z$ and integrating it along $\Gamma_{2}$, we have

$$
\int_{\varphi}^{\varphi_{5}} \frac{d s}{\left(s-\varphi_{2}\right) \sqrt{\varphi_{5}-s}}=-\sqrt{\frac{2}{3}} \operatorname{sign}(z) \xi .
$$

In (2.24), completing the integral yields (2.15).

(3) When $g=0$, the curves $\Gamma_{3}, \Gamma_{4}$ and $\Gamma_{5}$ have the following expressions:

$$
\begin{gathered}
\Gamma_{3}: z=\operatorname{sign}(z) \sqrt{\frac{2}{3}}\left(\varphi-\varphi_{6}\right) \sqrt{-\varphi}, \text { where } \varphi_{6}<\varphi \leq 0, \\
\Gamma_{4}: z=\operatorname{sign}(z) \sqrt{\frac{2}{3}}\left(\varphi_{6}-\varphi\right) \sqrt{-\varphi}, \text { where } \varphi<\varphi_{6}, \\
\Gamma_{5}: \quad z=\operatorname{sign}(z) \sqrt{\frac{2}{3}}\left(\varphi_{7}-\varphi\right) \sqrt{-\varphi}, \text { where } \varphi<0 .
\end{gathered}
$$

Substituting (2.25), (2.26) and (2.27) into $\frac{d \varphi}{d \xi}=z$ and integrating them along $\Gamma_{3}, \Gamma_{4}$ and $\Gamma_{5}$, respectively, it follows that

$$
\begin{gathered}
\int_{\varphi}^{0} \frac{d s}{\left(s-\varphi_{6}\right) \sqrt{-s}}=-\sqrt{\frac{2}{3}} \operatorname{sign}(z) \xi \quad\left(\operatorname{along} \Gamma_{3}\right), \\
\int_{-\infty}^{\varphi} \frac{d s}{\left(\varphi_{6}-s\right) \sqrt{-s}}=\sqrt{\frac{2}{3}} \operatorname{sign}(z) \xi \quad\left(\text { along } \Gamma_{4}\right),
\end{gathered}
$$




$$
\int_{\varphi}^{0} \frac{d s}{\left(\varphi_{7}-s\right) \sqrt{-s}}=-\sqrt{\frac{2}{3}} \operatorname{sign}(z) \xi \quad\left(\text { along } \Gamma_{5}\right) .
$$

In (2.28), (2.29) and (2.30), completing these integrals yields (2.16), (2.9) and (2.13) respectively.

(4) When $g_{1}(c)<g<0$, the curves $\Gamma_{6}$ and $\Gamma_{7}$ have the following expressions:

$$
\begin{aligned}
& \Gamma_{6}: z=\operatorname{sign}(z) \sqrt{\frac{2}{3}}\left(\varphi-\varphi_{9}\right) \sqrt{\varphi_{11}-\varphi}, \text { where } \varphi_{9}<\varphi \leq \varphi_{11}, \\
& \Gamma_{7}: \quad z=\operatorname{sign}(z) \sqrt{\frac{2}{3}}\left(\varphi_{10}-\varphi\right) \sqrt{\varphi_{12}-\varphi}, \text { where } 0<\varphi \leq \varphi_{12} .
\end{aligned}
$$

Substituting (2.31) and (2.32) into $\frac{d \varphi}{d \xi}=z$ and integrating them along $\Gamma_{6}$ and $\Gamma_{7}$ respectively, it follows that

$$
\begin{gathered}
\int_{\varphi}^{\varphi_{11}} \frac{d s}{\left(s-\varphi_{9}\right) \sqrt{\varphi_{11}-s}}=-\sqrt{\frac{2}{3}} \operatorname{sign}(z) \xi \quad\left(\text { along } \Gamma_{6}\right), \\
\int_{\varphi}^{\varphi_{12}} \frac{d s}{\left(\varphi_{10}-s\right) \sqrt{\varphi_{12}-s}}=-\sqrt{\frac{2}{3}} \operatorname{sign}(z) \xi \quad\left(\text { along } \Gamma_{7}\right) .
\end{gathered}
$$

In (2.33) and (2.34), completing these integrals yields (2.17) and (2.14) respectively.

(5) When $g=g_{1}(c)$, the curves $\Gamma_{8}, \Gamma_{9}$ and $\Gamma_{10}$ have the following expressions:

$$
\begin{gathered}
\Gamma_{8}: \quad z=\operatorname{sign}(z) \sqrt{\frac{2}{3}}\left(\varphi-\varphi_{13}\right) \sqrt{\varphi_{14}-\varphi}, \text { where } \varphi_{13}<\varphi \leq \varphi_{14}, \\
\Gamma_{9}: z=\operatorname{sign}(z) \sqrt{\frac{2}{3}}\left(\varphi_{13}-\varphi\right) \sqrt{\varphi_{14}-\varphi}, \text { where } \varphi<\varphi_{13}, \\
\Gamma_{10}: \quad z=\operatorname{sign}(z) \sqrt{\frac{2}{3}}\left(\varphi_{0}^{+}-\varphi\right) \sqrt{\left(\varphi_{0}^{+}-\varphi\right)}, \text { where } \varphi<\varphi_{0}^{+} .
\end{gathered}
$$

Substituting (2.35), (2.36) and (2.37) into $\frac{d \varphi}{d \xi}=z$ and integrating them along $\Gamma_{8}, \Gamma_{9}$ and $\Gamma_{10}$ respectively, it follows that

$$
\begin{gathered}
\int_{\varphi}^{\varphi_{14}} \frac{d s}{\left(s-\varphi_{13}\right) \sqrt{\varphi_{14}-s}}=-\sqrt{\frac{2}{3}} \operatorname{sign}(z) \xi \quad\left(\text { along } \Gamma_{8}\right), \\
\int_{-\infty}^{\varphi} \frac{d s}{\left(\varphi_{13}-s\right) \sqrt{\varphi_{14}-s}}=\sqrt{\frac{2}{3}} \operatorname{sign}(z) \xi \quad\left(\text { along } \Gamma_{9}\right), \\
\int_{-\infty}^{\varphi} \frac{d s}{\left(\varphi_{0}^{+}-s\right) \sqrt{\varphi_{0}^{+}-s}}=\sqrt{\frac{2}{3}} \operatorname{sign}(z) \xi \quad\left(\text { along } \Gamma_{10}\right) .
\end{gathered}
$$

In (2.38), (2.39) and (2.40), completing these integrals yields (2.18), (2.10) and (2.11) respectively. 


\section{Some soliton solutions of Eq. (1.1)}

Here we calculate the parametric representations of the smooth and nonsmooth soliton solutions for Eq. (1.1) by virtue of the formula (1.17). If we take $c=3, y=1, g_{1}=0$ and $g_{2}=-1$, i.e. $\xi=1-3 \tau$, our main results under different parametric conditions are listed in the following three propositions.

Proposition 3.1. Eq. (1.1) has the smooth soliton solution(see Fig. 2(a))

$$
\left\{\begin{array}{l}
u=\sqrt{1-\frac{9}{9+c \varphi_{1}(\xi)^{2}}} \\
x=\tau-\frac{1}{4}(-1+3 \tau)+\frac{1}{4} \arctan \eta_{6}
\end{array}\right.
$$

where $\eta_{6}=\frac{3(1-3 \tau)}{-3+(1-3 \tau)^{2}}$.
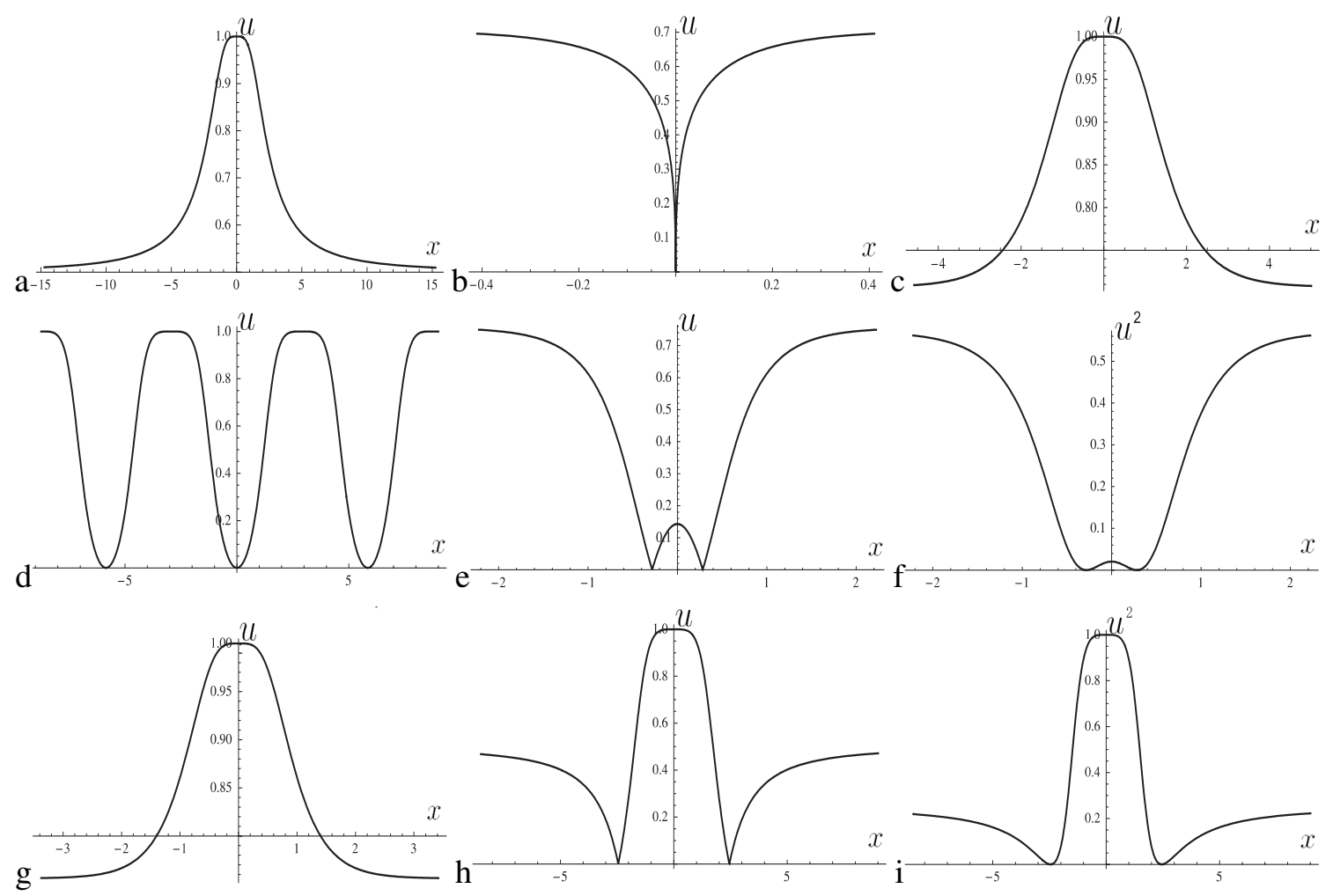

Fig. 2. The portraits of the soliton solutions of Eq. (1.1).

Proposition 3.2. Eq. (1.1) has peaked soliton solution(see Fig. 2(b))

$$
\left\{\begin{array}{l}
u=\sqrt{1-\frac{9}{9+c \varphi_{4}(\xi)^{2}}}, \\
x=\tau+\frac{1}{2} \frac{1}{3^{3 / 4}}\left(\arctan \left[-1+\sqrt{2} \tanh \eta_{7}\right]-\arctan \left[1+\sqrt{2} \tanh \eta_{7}\right]\right)-\frac{1}{2 \sqrt{2}} \frac{1}{3^{3 / 4}} \ln \left|\frac{-1+\tanh \eta_{7}}{1+\tanh \eta_{7}}\right|
\end{array}\right.
$$


the smooth soliton solution(see Fig. 2(c))

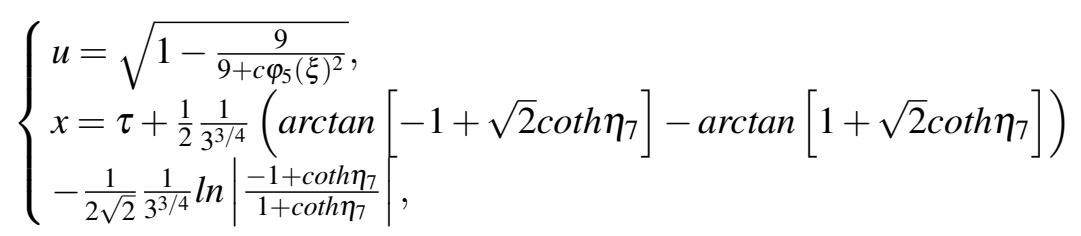

and the periodic solution(see Fig. 2(d))

$$
\left\{\begin{array}{l}
u=\sqrt{1-\frac{9}{9+c \varphi_{6}(\xi)^{2}}}, \\
x=\tau+\frac{\sqrt{2}}{2} \frac{1}{3^{3 / 4}} \arctan \left|\tan \eta_{7}\right|-\frac{1}{4} \frac{1}{3^{3 / 4}} \ln \left|\frac{-1+\sqrt{2} \tan \eta_{7}-\tan ^{2} \eta_{7}}{1+\sqrt{2} \tan \eta_{7}+\tan ^{2} \eta_{7}}\right|,
\end{array}\right.
$$

where $\eta_{7}=\frac{1-3 \tau}{\sqrt{2} 3^{1 / 4}}$.

Proposition 3.3. Eq. (1.1) has the novel W-shaped soliton solution(see Fig. 2(e))

$$
\left\{\begin{array}{l}
u=\sqrt{1-\frac{9}{9+c \varphi_{9}(\xi)^{2}}} \\
x=\frac{1}{14}\left(8 \tau-\sqrt{2} \arctan \left[\frac{1}{3 \sqrt{3}} \operatorname{sech}\left[3 \sqrt{\frac{3}{2}} \tau\right]\left(-6 \cosh \eta_{8}-8 \sinh \eta_{8}+\sinh \left[3 \sqrt{\frac{3}{2}} \tau\right]\right)\right]\right) \\
-\sqrt{2}\left(\arctan \left[\frac{1}{3 \sqrt{3}} \operatorname{sech}\left[3 \sqrt{\frac{3}{2}} \tau\right]\left(6 \cosh \eta_{8}-8 \sinh \eta_{8}+\sinh \left[3 \sqrt{\frac{3}{2}} \tau\right]\right)\right]\right),
\end{array}\right.
$$

the smooth soliton solution(see Fig. 2(g))

$$
\left\{\begin{array}{l}
u=\sqrt{1-\frac{9}{9+c \varphi_{10}(\xi)^{2}}}, \\
x=\frac{1}{14}\left(8 \tau-\sqrt{2} \arctan \left[\frac{1}{3 \sqrt{3}} \operatorname{sech}\left[3 \sqrt{\frac{3}{2}} \tau\right]\left(-6 \cosh \eta_{8}+8 \sinh \eta_{8}+\sinh \left[3 \sqrt{\frac{3}{2}} \tau\right]\right)\right]\right) \\
-\sqrt{2}\left(\arctan \left[\frac{1}{3 \sqrt{3}} \operatorname{sech}\left[3 \sqrt{\frac{3}{2}} \tau\right]\left(6 \cosh \eta_{8}+8 \sinh \eta_{8}+\sinh \left[3 \sqrt{\frac{3}{2}} \tau\right]\right)\right]\right),
\end{array}\right.
$$

the novel W-shaped soliton solution(see Fig. 2(h))

$$
\left\{\begin{array}{l}
u=\sqrt{1-\frac{9}{9+c \varphi_{11}(\xi)^{2}}} \\
x=\tau-\frac{1}{4}(-1+3 \tau)-\frac{1}{8} \ln \left|\frac{3+3(1-3 \tau)+(1-3 \tau)^{2}}{3-3(1-3 \tau)+(1-3 \tau)^{2}}\right|
\end{array}\right.
$$

where $\eta_{8}=\sqrt{\frac{3}{2}}(2-3 \tau)$.

Remark 3.1. (1) The reason that the cuspidal points arise is the square root of the function $u^{2}$, while on $x-u^{2}$ plane, the soliton solutions are smooth (see Figs. 2(f, i)).

(2) Like the other functions $\varphi_{2}(\xi), \varphi_{3}(\xi), \varphi_{7}(\xi)$ and $\varphi_{8}(\xi)$, we can't finish the indefinite integrals $x=\int u^{2} d \tau$. Therefore, we couldn't give the parametric representations of these solutions of Eq. (1.1) which needs to deal with further.

\section{Conclusions}

In this paper, based on the relationship of the solutions between the Novikov equation and Eq. (1.2), we derived the parametric representations of the soliton solutions for the Novikov equation. 
We obtained the traveling wave solutions of Eq. (1.2) by adopting the bifurcation method of dynamical systems. Further, in terms of the formula (1.17), we obtained the parametric representations of the smooth and nonsmooth soliton solutions of the Novikov equation. From the above analysis, we compare our results with previous studies through the soliton solutions and their research techniques.

1. Matsuno [7] transformed the Novikov equation to Eq. (1.2) by the reciprocal transformation and revealed that the smooth multisoliton solutions can be solved in terms of the tau-function associated with the $N$-soliton solution of a model equation [15], whose modified versions are obtained by the reciprocal transformations [16, 17]. By means of the traveling wave solutions of Eq. (1.2) and combining the formula (1.17), we investigated the smooth and nonsmooth soliton solutions of the Novikov equation which include peaked soliton, smooth soliton, W-shaped soliton and periodic solutions.

2. Li [8] obtained cuspons and compactons of the Novikov equation by applying the bifurcation method of dynamical systems to directly. While we discussed Eq. (1.2) by using the same method and derived its solitary wave, blow-up wave and periodic wave solutions. Some novel soliton solutions of the Novikov equation obtained in this paper didn't find in [7, 8]. Therefore, our results extend the previous studies.

In this sense, we partly enrich the properties of the Novikov equation and demonstrate the applicability of the proposed approach. To the best of our knowledge, this is probably the first time to discuss these novel soliton solutions of the Novikov equation.

\section{Acknowledgements}

The authors thank the handling editor and the reviewers for their valuable comments and suggestions, which improved the completeness of the paper. Thank you very much for your time and patience. This work is supported by the National Natural Science Foundation of China (No.11171115 and 11361069).

\section{References}

[1] V. Novikov, Generalizations of the Camassa-Holm equation, J. Phys. A: Math. Theor. 42 (2009) 342002.

[2] A.N.W. Hone and J. Wang, Integrable peakon equations with cubic nonlinearity, J. Phys. A: Math. Theor. 41 (2008) 372002.

[3] N. Li and Q. Liu, On bi-Hamiltonian structure of two-component Novikov equation, Phys. Lett. A 377 (2013) 257-261.

[4] X. Geng and B. Xue, An extension of integrable peakon equations with cubic nonlinearity, Nonlinearity 22 (2009) 1847-1856.

[5] A.N.W. Hone, H. Lundmark and J. Szmigielski, Explicit multipeakon solutions of Novikov's cubically nonlinear integrable Camassa-Holm type equation, Dyn. Partial Differ. Equ. 6 (2009) 253-289.

[6] K. Grayshan, Peakon solutions of the Novikov equation and properties of the data-to-solution map, $J$. Math. Anal. Appl. 397 (2013) 515-521.

[7] Y. Matsuno, Smooth multisoliton solutions and their peakon limit of Novikovs Camassa-Holm type equation with cubic nonlinearity, J. Phys. A: Math. Theor. 46 (2013) 365203.

[8] J. Li, Exact Cuspon and Compactons of the Novikov equation, Int. J. Bifurcat. Chaos 24 (2014) 1450037.

[9] Z. Liu and T. Qian, Peakons and their bifurcation in a generalized Camassa-Holm equation, Int. J. Bifurcat. Chaos 11 (2001) 781-792.

[10] Z. Liu and C. Yang, The application of bifurcation method to a higher-order KdV equation, J. Math. Anal. Appl. 275 (2002) 1-12. 
[11] C. Pan, L. Ling and Z. Liu, A new integrable equation with cuspons and periodic cuspons, Phys. Scr. 89 (2014) 105207.

[12] J. Li and G. Chen, On a class of singular nonlinear traveling wave equations, Int. J. Bifurcat. Chaos 17 (2007) 4049-4065.

[13] J. Li and Z. Qiao, Bifurcations of traveling wave solutions for an integrable equation, J. Math. Phys. 51 (2010) 042703.

[14] P. G. Estévez and S. Leble, A wave equation in $2+1$ : Painlevé analysis and solutions, Inv. Prob. 11 (1995) 925-937.

[15] R. Hirota and Satsuma, N-soliton solutions of model equations for shallow water waves, J. Phys. Soc. Japan 40 (1976) 611-612.

[16] P. G. Estévez and J. Prada, Singular manifold method for an equation in $2+1$ dimensions, J. Nonlinear Math. Phys. 12 (2005) 266-279.

[17] P. G. Estévez, Reciprocal transformations for a spectral problem in $2+1$ dimensions, Theor. Math. Phys. 159 (2009) 762-768. 